Supporting information to

\title{
Quantification of sudden light-induced polarization in bacteriorhodopsin by optical rectification
}

\author{
A. Colonna, G. I. Groma, J.-L. Martin, M. Joffre, M. H. Vos
}

Here we discuss infrared generation in the femtosecond regime in a dispersive bulk non linear medium whith thickness e $>>\lambda_{e x}, \lambda_{e m}$ (excitation and emission wavelength respectively), $\frac{n_{v} c T}{\Delta n}$, (with $\mathrm{T}$ the visible pulse duration at the entrance, $\Delta \mathrm{n}$ the visible refractive index dispersion, $\mathrm{n}_{v}$ the visible refractive index), under non phase matching conditions.

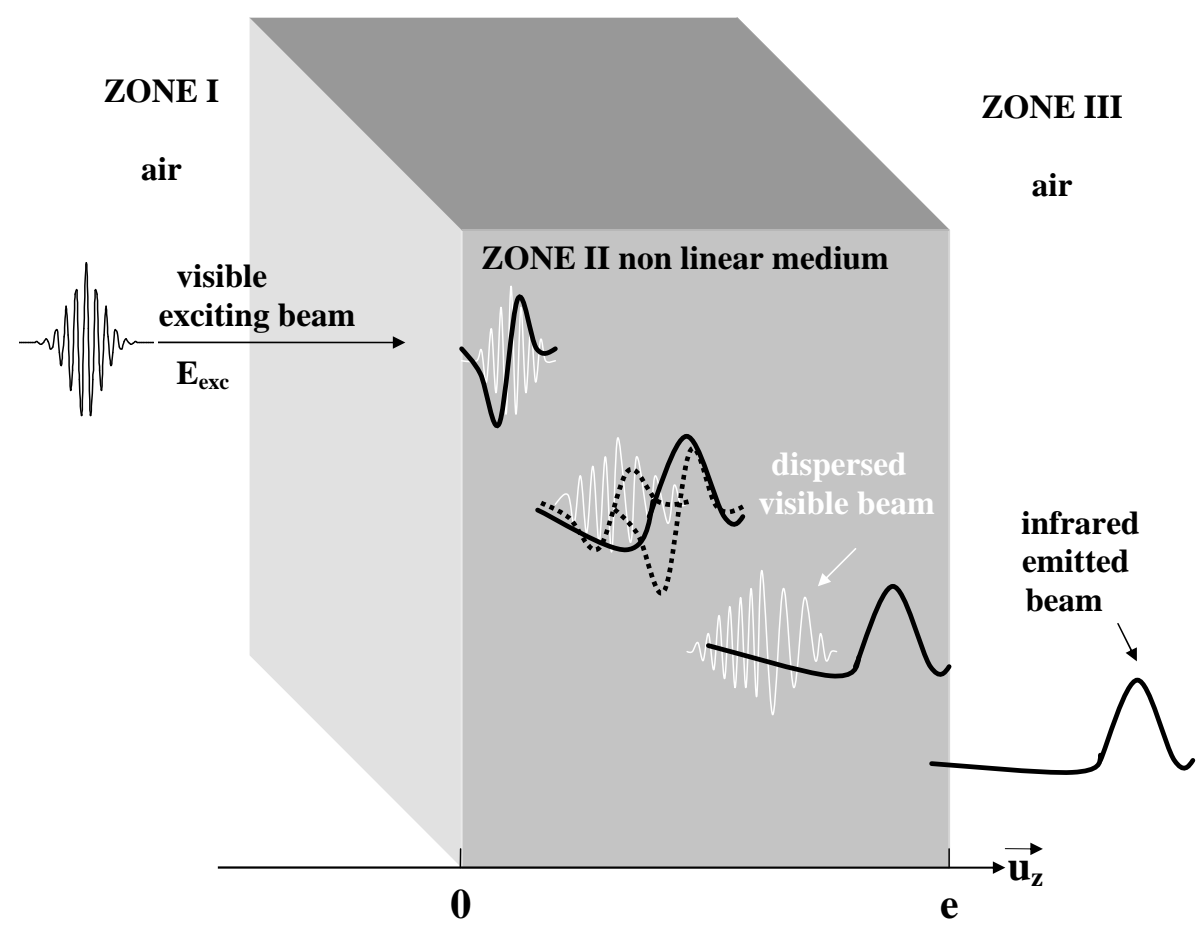

FIG. 1 : Infrared emission generated in a thick non linear medium with no phase matching.

We first describe the phenomenon qualitatively. As seen in figure 1, upon entering the nonlinear medium, the visible excitation beam starts to interact and generates a second order polarization $\mathrm{P}^{(2)}(\mathrm{t})$. In the plane wave approximation, the infrared (IR) emitted beam is proportional to the first derivative of the polarization. The visible and infrared beams propagate through the medium at different speeds with the visible pulse lagging behind the infrared pulse. While propagating through the medium, the visible excitation beam continues generating infrared, the leading edge of which interferes destructively with the trailing edge of the IR beam generated close to the entrance surface. In case of a non-dispersive medium, two time-delayed half-cycle pulses are generated, resulting of the contribution of both entrance and exit sides [S1,S2]. In our case the medium group velocity dispersion broadens the visible pulse which becomes too long 
to produce infrared radiation through optical rectification at the exit side. Hence, the exit-side contribution vanishes. Taking into account the transmission factor at normal incidence [S3], the half cycle thus created can be written as :

$$
E_{I R}(t)=\frac{1+n_{v}}{\epsilon_{o}\left(n_{v}+n_{I R}\right)\left(1+n_{I R}\right)} \frac{L_{e f f}}{c T} P^{(2)}(t)
$$

where $\mathrm{n}_{i r}$ is the infrared refractive index, and $\mathrm{L}_{\text {eff }}$ the effective length of the IR generation $\left(\mathrm{L}_{e f f}=\frac{c T}{n_{v}-n_{I R}}\right)$.

The nonlinear polarization generated at the entrance side is instantaneous and can be written as :

$$
\begin{array}{r}
P^{(2)}(t)=\epsilon_{o} \chi^{(2)} \frac{|\underline{E}(t)|^{2}}{2} \\
\text { and } \quad E_{I R}(t)=\frac{\left(1+n_{v}\right) L_{e f f} \chi^{(2)}|\underline{E}(t)|^{2}}{2 c T\left(n_{v}+n_{I R}\right)\left(1+n_{I R}\right)}
\end{array}
$$

As the visible pulse temporally broadens during propagation through the medium, effective IR generation occurs only on a limited thickness, and the net infrared output beam is described by equation 2 .

In the following we will give a more formal treatment of the phenomenon. Consider an excitation beam described by a complex electric field $\underline{E}(\mathrm{z}, \omega)=A(z, \omega) \mathrm{e}^{i k(\omega) z}$ where $A(z, \omega)$ is the pulse envelope, $\omega$ the frequency and $\mathrm{k}$ the wave number. A nonlinear polarization $\mathrm{P}^{N L}$ is created in the medium.

The Maxwell equation can be written :

$$
\frac{\partial^{2} A(z, \omega)}{\partial z^{2}} e^{i k(\omega) z}-2 i k(\omega) \frac{\partial A}{\partial z} e^{i k(\omega) z}=-\mu_{o} \omega^{2} \underline{P}^{(N L)}(z, \omega)
$$

and in the paraxial approximation $\left(\left|\frac{\partial^{2} A(z, \omega)}{\partial z^{2}}\right|<<\left|\frac{\partial A(z, \omega)}{\partial z}\right|\right)$ :

$$
\frac{\partial \underline{A}(z, \omega)}{\partial z}=\frac{i \mu_{o} c}{2 n(\omega)} \omega \underline{P}^{(N L)}(z, \omega)
$$

As $\frac{\partial \underline{E}}{\partial z}=\frac{\partial A(z, \omega)}{\partial z}+i k(\omega) A(z, \omega) \mathrm{e}^{i k(\omega) z}$, equation 4 can be written as :

$$
\frac{\partial \underline{E}(z, \omega)}{\partial z}-i k(\omega) \underline{E}(z, \omega)=\frac{i \mu_{o} c}{2 n(\omega)} \omega \underline{P}^{(N L)}(z, \omega)
$$

The IR beam depends on medium non linearity and dispersion properties. To consider both of 
them separately, we write

$$
\underline{E}(z, t)=A(z, t) e^{\left(i k_{o} z-\omega_{o} t\right)}
$$

and in the frequency domain :

$$
\underline{E}(z, \omega)=A\left(z, \omega-\omega_{o}\right) e^{i k_{o} z}
$$

where $\mathrm{A}(\mathrm{z}, \mathrm{t})$ is the field envelope, $\omega_{o}$ the carrier frequency and $k_{o}$ the wave vector. After taking the derivative and writing $\omega-\omega_{0} \rightarrow \omega_{o}$ we get :

$$
\frac{\partial A(z, \omega)}{\partial z}=\underbrace{i\left(k\left(\omega+\omega_{o}\right)-k_{o}\right) A(z, \omega)}_{\text {dispersive }}+\underbrace{\frac{i \mu_{o} c}{2 n(\omega)} \omega \underline{P}_{\text {effects }}^{(N)}(z, \omega)}_{\text {nonlinear }}
$$

This propagation equation is written in the form

$$
\frac{\partial A(z, \omega)}{\partial z}=(\hat{D}+\hat{N}) A(z, \omega)
$$

with $\hat{D}$ the linear (temporal dispersion) and $\hat{N}$ the nonlinear (optical rectification in our case) operators respectively. To solve this problem numerically, we use the split-step method (figure 2), based on partition of the medium into discrete spatial steps. In alternating steps, linear and nonlinear operators are applied separetely.

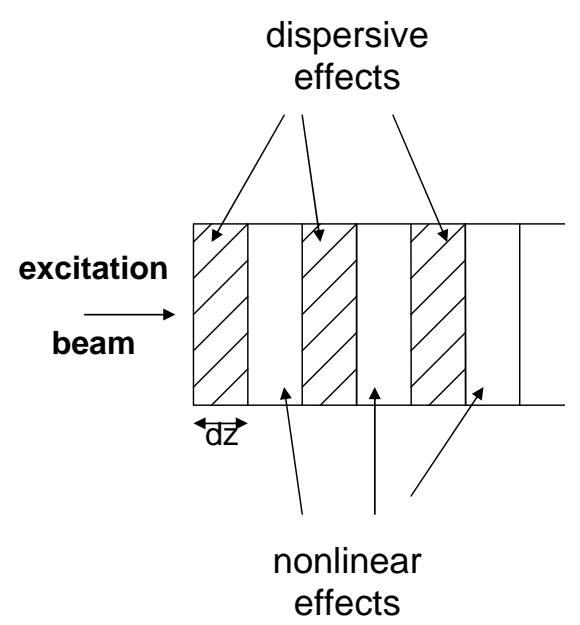

FIG. 2 : Split-Step method : schematic principle. 
Let us calculate nonlinear and dispersion effects on the visible beam :

1. Dispersion effects are calculated in the frequency domain :

$$
\begin{array}{r}
\frac{\partial A(z, \omega)}{\partial z}=i\left(k\left(\omega+\omega_{o}\right)-k_{o}\right) A(z, \omega) \\
\text { or } A(z, \omega)=\exp \left(i\left(k\left(\omega+\omega_{o}\right)-k_{o}\right) z\right) A(0, \omega)
\end{array}
$$

2. Nonlinear effects :

$$
A(z+d z, \omega)=A(z, \omega)+\frac{i \mu_{o} c d z}{2 n(\omega)} \omega \underline{P}^{N L}(z, \omega)
$$

We restrain ourselves to second order non linearities and only keep terms corresponding to optical rectification, considering our experimental conditions (type II crystal). Polarization generated in the nonlinear medium excited by the femtosecond visible beam is

$$
\begin{array}{r}
P^{(2)}(z, t)=\frac{\epsilon_{o} \chi^{(2)}}{2}\left|\underline{E}_{e}+\underline{E}_{o}+c . c\right|^{2} \\
=\epsilon_{o} \chi^{(2)}\left[\underline{E}_{e}(z, t) \underline{E}_{o}(z, t)^{*}+c . c\right]
\end{array}
$$

Using equation 11:

$$
\underline{E}(z+d z)=\underline{E}(z, \omega)+\frac{i \omega d z}{2 n(\omega) \epsilon_{o} c} \underline{P}^{(2)}(z, \omega)
$$

The effect of third order non linearities effects (two photon absorption and Kerr effect) have also been explored but appear to be negligible in our experimental conditions.

In this way we have calculated the infrared beam generated by an $\mathrm{AgGaS}_{2}$ (Silver Thiogallate) crystal, used in our experiments. The spectral profile of the simulated beam is in very good agreement with the physical explanation (figure 3) : it is proportional to the polarization created in the medium. 


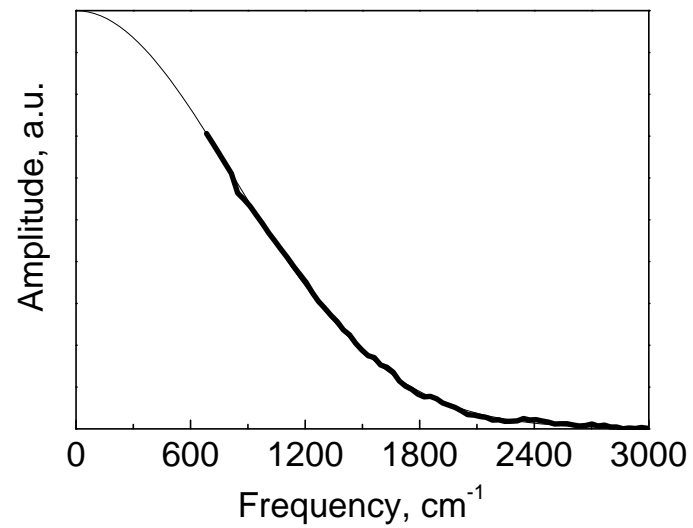

FIG. 3 : Thin line : polarization spectrum created by a gaussian exciting pulse, $11 \mathrm{fs}$ duration, in the instantaneouse case. Bold line : emitted beam spectrum generated by a $1 \mathrm{~mm}$-thick $\mathrm{AgGaS}_{2}$ crystal modelized using the split-step method (using 800 steps). The sample is excited by a gaussian pulse, 11 fs duration, centered at $560 \mathrm{~nm}$. The $\mathrm{AgGaS}_{2}$ emitted beam is proportional to the polarization created in the medium.

\section{Bibliography}

[S1] L. Xu, X.C. Zhang, D. Auston, Appl. Phys. Lett. 61, 1784 (1992)

[S2] K. Wynne,J. Carey, J. Opt. Comm. 256, 400 (2005)

[S3] A. Bonvalet, PhD Thesis, University of Paris XI, Orsay (1997) 